\title{
Theoretical foundations of digital vector Fourier analysis of two-dimensional signals padded with zero samples
}

\author{
O. V. Ponomarevaa, Dr. Sc., Tech., Professor, orcid.org/0000-0002-7311-3108, ponva@mail.ru \\ A. V. Ponomareva, PhD, Econ., Associate Professor, orcid.org/0000-0002-3746-9289 \\ aKalashnikov Izhevsk State Technical University, 7, Studencheskaya St., 426069, Izhevsk, Russian Federation
}

\begin{abstract}
Introduction: The practice of using Fourier-processing of finite two-dimensional signals (including images), having confirmed its effectiveness, revealed a number of negative effects inherent in it. A well-known method of dealing with negative effects of Fourier-processing is padding signals with zeros. However, the use of this operation leads to the need to provide information control systems with additional memory and perform unproductive calculations. Purpose: To develop new discrete Fourier transforms for efficient and effective processing of two-dimensional signals padded with zero samples. Method: We have proposed a new method for splitting a rectangular discrete Fourier transform matrix into square matrices. The method is based on the application of the modulus comparability relation to order the rows (columns) of the Fourier matrix. Results: New discrete Fourier transforms with variable parameters were developed, being a generalization of the classical discrete Fourier transform. The article investigates the properties of Fourier transform bases with variable parameters. In respect to these transforms, the validity has been proved for the theorems of linearity, shift, correlation and Parseval's equality. In the digital spectral Fourier analysis, the concepts of a parametric shift of a two-dimensional signal, and a parametric periodicity of a two-dimensional signal have been introduced. We have estimated the reduction of the required memory size and the number of calculations when applying the proposed transforms, and compared them with the discrete Fourier transform. Practical relevance: The developed discrete Fourier transforms with variable parameters can significantly reduce the cost of Fourier processing of two-dimensional signals (including images) padded with zeros.
\end{abstract}

Keywords - discrete Fourier transform, two-dimensional signal, Fourier processing, effects of discrete Fourier transform, basis, variable parameter.

For citation: Ponomareva O. V., Ponomarev A. V. Theoretical foundations of digital vector Fourier analysis of two-dimensional signals padded with zero samples. Informatsionno-upravliaiushchie sistemy [Information and Control Systems], 2021, no. 1, pp. 55-65. doi:10.31799/1684-8853-2021-1-55-65

\section{Introduction}

Fourier-processing of finite discrete two-dimensional (FDTD) signals (including images) in informational control (IC) systems is the most important method for studying processes and analyzing information [1-8]. The theoretical basis of Fourierprocessing of FDTD signals is two-dimensional direct and inverse discrete Fourier transforms (2D DFT, 2D IDFT) [9-15] which can be represented in form of:

- algebraic form $2 \mathrm{D}$ DFT

$$
\begin{gathered}
S_{N_{1}, N_{2}}\left(k_{1}, k_{2}\right)= \\
=\frac{1}{N_{1} N_{2}} \sum_{n_{1}=0}^{N_{1}-1} \sum_{n_{2}=0}^{N_{2}-1} x\left(n_{1}, n_{2}\right) W_{N_{1}}^{k_{1} n_{1}} \cdot W_{N_{2}}^{k_{2} n_{2}},
\end{gathered}
$$

where $S_{N_{1}, N_{2}}\left(k_{1}, k_{2}\right)$ are coefficients (bins) 2D $\mathrm{DFT} ; \quad k_{1}=\overline{0,\left(N_{1}-1\right)}, \quad k_{2}=\overline{0,\left(N_{2}-1\right)}$ are spatial frequencies; $x\left(n_{1}, n_{2}\right)$ is $2 \mathrm{D}$ signal; $n_{1}=\overline{0, N_{1}-1}$,

$$
\begin{aligned}
& n_{2}=\overline{0, N_{2}-1 ;} \quad W_{N_{1}}^{k_{1} n_{1}}=\exp \left(-j \frac{2 \pi}{N_{1}} k_{1} n_{1}\right) ; \quad W_{N_{2}}^{k_{2} n_{2}}= \\
& =\exp \left(-j \frac{2 \pi}{N_{2}} k_{2} n_{2}\right)
\end{aligned}
$$

- algebraic form 2D IDFT

$$
x\left(n_{1}, n_{2}\right)=\sum_{k_{1}=0}^{N_{1}-1} \sum_{k_{2}=0}^{N_{2}-1} S_{N_{1}, N_{2}}\left(k_{1}, k_{2}\right) W_{N_{1}}^{-k_{1} n_{1}} \cdot W_{N_{2}}^{-k_{2} n_{2}} .
$$

The practice of using DFT and 2D DFT, on the one hand, confirmed their efficiency, on the other hand, revealed a number of effects: aliasing effect, scalloping effect, picket fence effect, negatively affecting on the results of analysis and information processing [16-21].

To eliminate these negative effects of DFT and 2D DFT, the zero-padding operation (ZPO) the FDTD signal has found wide application. ZPO can significantly reduce the impact of negative effects on the results of Fourier-processing [22, 23]. However, effective use of ZPO requires solving the problem of Fourier-processing of FDTD of this kind of signals. The essence of the problem lies in the fact that in Fourier-processing of signals subjected to ZPO, on the one hand, it is necessary to provide the corresponding IC systems with a significant amount of additional memory, on the other hand, the IC systems must perform unproductive computations with zero samples, which significantly increases time of Fourier-processing. The paper pro- 
poses and investigates new discrete Fourier transforms, which allow efficient and effective analysis and processing of two-dimensional signals padded with zero samples.

\section{The role of the zero-padding operation of FDTD signals in two-dimensional Fourier-processing}

The systems analysis of Fourier-processing theory of FDTD signals made it possible to formulate its axiomatic basic provisions:

- determination of FDTD signals on a finite two-dimensional reference plane, which is interpreted as a two-dimensional fundamental period $S A_{N_{1} \times N_{2}}$ (2D period). 2D period is set by horizontal and vertical periods;

- determination of the shift of a two-dimensional discrete signal in the form of a cyclic shift, carried out by cyclic permutation of its samples on the final reference area $S A_{N_{1} \times N_{2}}$;

- definition of a complete two-dimensional basis system

$$
\operatorname{def}_{N_{1}, N_{2}}\left(k_{1}, n_{1}, k_{2}, n_{2}\right)=W_{N_{1}}^{k_{1} n_{1}} \cdot W_{N_{2}}^{k_{2} n_{2}},
$$

where $n_{1}=\overline{0, N_{1}-1} ; \quad n_{2}=\overline{0, N_{2}-1} ; \quad k_{1}=\overline{0,\left(N_{1}-1\right)}$; $k_{2}=\overline{0,\left(N_{2}-1\right)}$.

As a result of the discreteness and periodicity of $2 \mathrm{D}$ signals in the spatial domain, the periodicity and discreteness of 2D Fourier spectra in the spatial-frequency domain, the mathematical operations of convolution and correlation are cyclical. However, the analysis, design, and modeling of isoplanatic systems requires the results of linear operations with $2 \mathrm{D}$ signals.

The method, which allows obtaining the results of linear operations using cyclic operations, consists in expanding the reference regions with zero samplex of the convoluted signals by applying ZPO to them.

If the reference area $S A_{V_{1} \times V_{2}}$ of signal $x\left(n_{1}, n_{2}\right)$ and the reference area $S A_{Q_{1} \times Q_{2}}$ of signal $y\left(n_{1}, n_{2}\right)$ are specified, then the size of the reference area, padded with zeros to obtain linear convolution $h_{\text {linear }}\left(n_{1}, n_{2}\right)$, should be

$$
S A_{\left(V_{1}+Q_{1}\right) \times\left(V_{2}+Q_{2}\right)},
$$

where $n_{1}=\overline{0,\left(V_{1}+Q_{1}-1\right)} ; n_{2}=\overline{0,\left(V_{2}+Q_{2}-1\right)}$.

And the size of the reference area for obtaining linear correlation $C_{L}\left(n_{1}, n_{2}\right)$ should be

$$
S A_{2 V_{1} \times 2 V_{2}}
$$

where $n_{1}=\overline{0,\left(2 V_{1}-1\right)} ; n_{2}=\overline{0,\left(2 V_{2}-1\right)}$.
Therefore, the algorithm for obtaining 2D linear convolution based on 2D cyclic convolution consists of the following operations:

1. Pad $2 \mathrm{D}$ signals $x\left(n_{1}, n_{2}\right)$ and $y\left(n_{1}, n_{2}\right)$ with $Q_{1}$, $Q_{2}$ and $V_{1}, V_{2}$ zero samples respectively, which sets new $2 \mathrm{D}$ signals $x_{0}\left(n_{1}, n_{2}\right), y_{0}\left(n_{1}, n_{2}\right)$ with horizontal $N_{2}$ and vertical $N_{1}$ periods according to the ratios

$$
N_{1} \geq\left(V_{1}+Q_{1}-1\right) ; N_{2} \geq\left(V_{2}+Q_{2}-1\right) \text {. }
$$

2. Perform 2D DFT of 2D signals $x_{0}\left(n_{1}, n_{2}\right)$ and $y_{0}\left(n_{1}, n_{2}\right)$ :

$$
\begin{aligned}
& x_{0}\left(n_{1}, n_{2}\right) \stackrel{F}{\longrightarrow} X_{0, N_{1}, N_{2}}\left(k_{1}, k_{2}\right) ; \\
& y_{0}\left(n_{1}, n_{2}\right) \stackrel{F}{\longrightarrow} Y_{0, N_{1}, N_{2}}\left(k_{1}, k_{2}\right),
\end{aligned}
$$

where $\stackrel{F}{\longrightarrow}$ is the 2D DFT execution symbol.

3. Perform 2D IDFT product

$$
X_{0, N_{1}, N_{2}}\left(k_{1}, k_{2}\right) \cdot Y_{0, N_{1}, N_{2}}\left(k_{1}, k_{2}\right) \text {. }
$$

The algorithm for obtaining a linear 2D correlation function based on a cyclic 2D correlation function is easy to obtain from the previous algorithm. Fig. 1, $a$ and $b$ illustrates the differences between cyclic $C_{C}\left(n_{1}, n_{2}\right)$ and linear $C_{L}\left(n_{1}, n_{2}\right)$ correlation functions of a finite unit $2 \mathrm{D}$ signal.

According to the two-dimensional version of the Wiener - Khinchin theorem, Fourier transform of the linear 2D correlation function allows one to obtain the energy spectrum of a $2 \mathrm{D}$ signal. There is a so-called direct method for obtaining the energy spectrum of a $2 \mathrm{D}$ signal $x\left(n_{1}, n_{2}\right)$, bypassing the stage of obtaining the correlation function:

$$
G_{N_{1}, N_{2}}\left(k_{1}, k_{2}\right)=N_{1} N_{2}\left|S_{N_{1}, N_{2}}\left(k_{1}, k_{2}\right)\right|^{2} .
$$

A significant drawback of this definition of the energy spectrum of a $2 \mathrm{D}$ signal $x\left(n_{1}, n_{2}\right)$ is insufficient detailing $G_{N_{1}, N_{2}}\left(k_{1}, k_{2}\right)$, for example, to fulfill the conditions of Pugachev canonical signal decomposition. The method of increasing the detail $G_{N_{1}, N_{2}}\left(k_{1}, k_{2}\right)$ is carried out by padding the $2 \mathrm{D}$ signal $x\left(n_{1}, n_{2}\right)$ with zeros at least twice. Fig. $2, a$ and $b$ illustrates the detailing of the energy spectrum of a finite single $2 \mathrm{D}$ signal.

As noted in the introduction, the effective application of the ZPO requires a solution to the problem of Fourier-processing of FDTD signals padded with zero samples. The essence of the problem lies in the fact that in Fourier-processing of signals subjected to the ZPO, on the one hand, it is necessary to provide the corresponding IC system with a significant additional amount of RAM (storage), on the other hand, IC system must perform a lot of non-productive calculations with zero samples, which significantly increases the time of Fourier-processing. 
a)

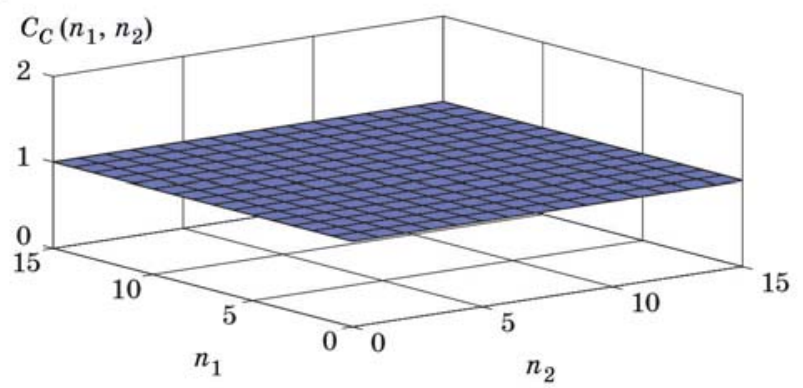

b)

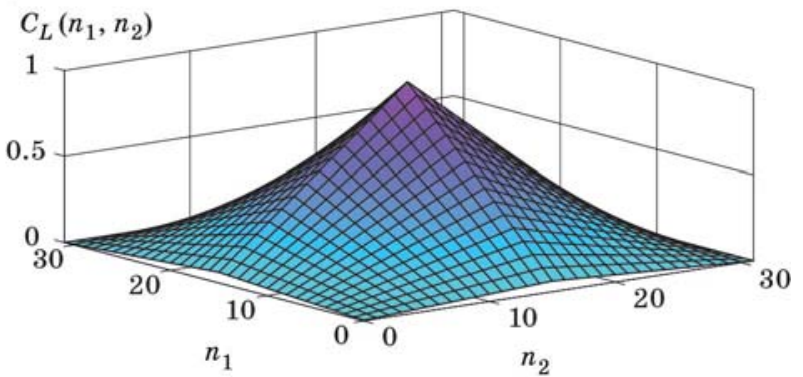

Fig. 1. Cyclic $(a)$ and linear $(b)$ correlation $2 \mathrm{D}$ functions of a finite single 2D signal
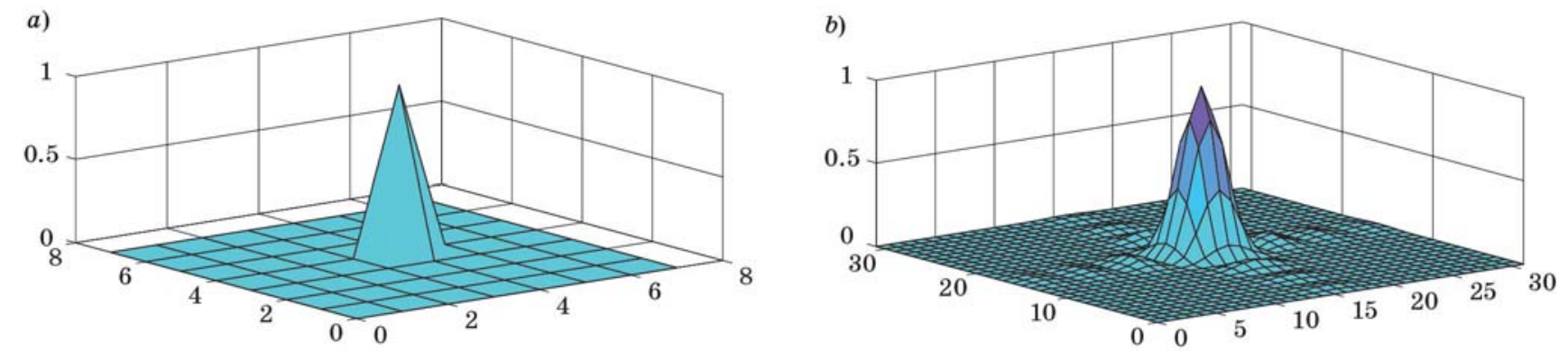

Fig. 2. Energy spectrum of a finite single 2D signal with zero frequency in the center of the spectrum: $a-$ a finite single $2 \mathrm{D}$ signal $N_{1}=8, N_{2}=8 ; b-$ a finite single $2 \mathrm{D}$ signal, padded with zero samples to $N_{1}=32, N_{2}=32$

Let us consider a generalization of 2D DFT in the form of a 2D DFT with a variable parameter, which makes it possible to efficiently analyze and process two-dimensional signals subjected to ZPO.

\section{Two-dimensional DFT with variable parameter}

Let two 2D signals be given: a signal $\mathbf{X}_{N_{1} \times N_{2}}$ and a signal $\mathbf{O}_{N_{1} \times N_{2}}$ with zero samples.

To perform the linear transformations considered in the previous section, it is necessary to pad (supplement) the horizontal period of the $2 \mathrm{D}$ signal $\mathbf{X}_{N_{1} \times N_{2}}$ with $\left(r_{2}-1\right)$ zero matrices $\mathbf{O}_{N_{1} \times N_{2}}$, which leads to a block matrix:

$$
\mathbf{X}_{N_{1} \times\left(N_{2} r_{2}\right)}=\left[\begin{array}{ccccc}
0 & 1 & \cdot & \cdot & \left(r_{2}-1\right) \\
\mathbf{X}_{N_{1} \times N_{2}} & \mathbf{O}_{N_{1} \times N_{2}} & \cdot & \cdot & \mathbf{O}_{N_{1} \times N_{2}}
\end{array}\right] \cdot
$$

Taking into account the separability property of the 2D DFT, Fourier transform of a signal $\mathbf{X}_{N_{1} \times\left(N_{2} r_{2}\right)}$ in matrix form can be represented as

$$
\mathbf{S}_{N_{1} \times\left(N_{2} r_{2}\right)}^{k_{1}, k_{2}}=\frac{1}{N_{1} N_{2}} \mathbf{F}_{N_{1} \times N_{1}}^{(2)}\left[\mathbf{X}_{N_{1} \times\left(N_{2} r_{2}\right)} \mathbf{F}_{\left(N_{2} r_{2}\right) \times\left(N_{2} r_{2}\right)}^{(1)}\right],
$$

where

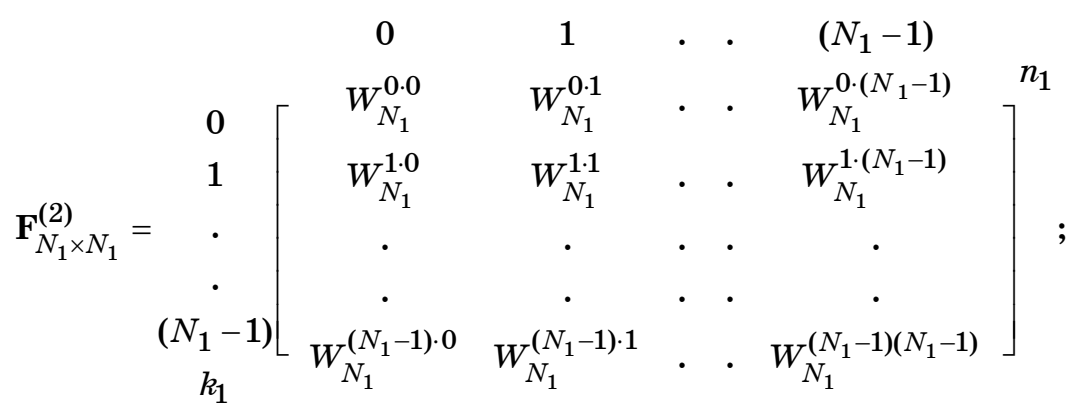




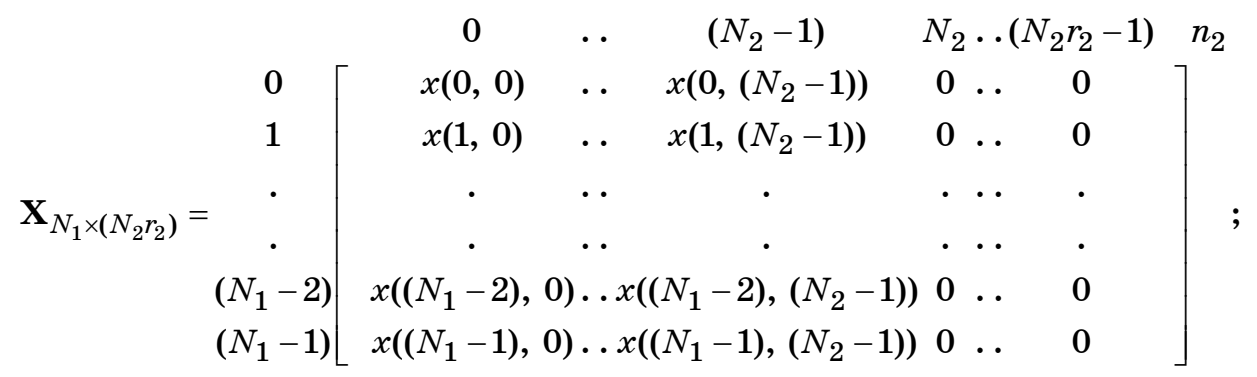

$$
\begin{aligned}
& n_{1}
\end{aligned}
$$

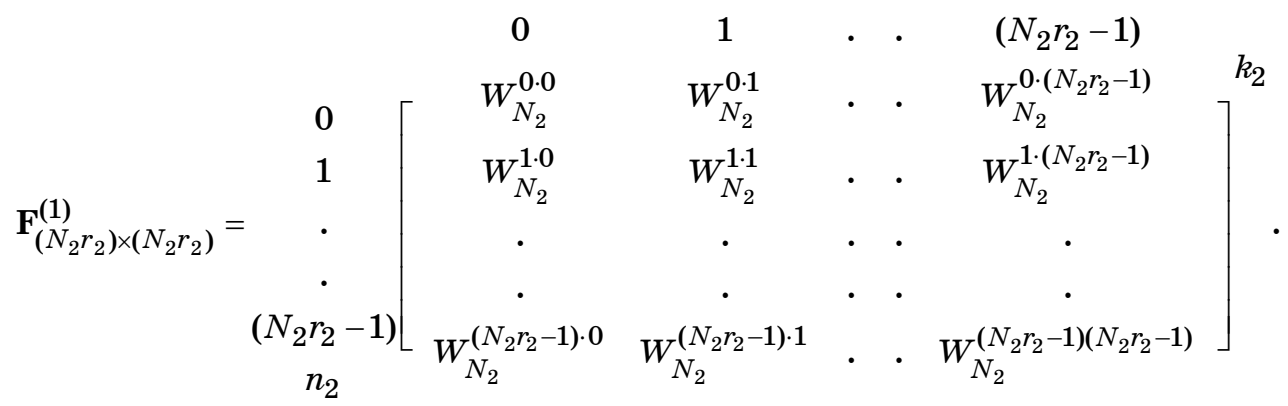

Let us interrogate the structure of matrix equation (2). It is easy to see that the multiplication of matrices $\mathbf{X}_{N_{1} \times\left(N_{2} r_{2}\right)}$ and $\mathbf{F}_{\left(N_{2} r_{2}\right) \times\left(N_{2} r_{2}\right)}^{(1)}$ leads to a rectangular matrix $\mathbf{Y}_{N_{1} \times\left(N_{2} r_{2}\right)}$. A matrix $\mathbf{Y}_{N_{1} \times\left(N_{2} r_{2}\right)}$ can be interpreted as the product of a matrix $\mathbf{X}_{N_{1} \times N_{2}}$ by a matrix $\mathbf{F}_{N_{2} \times\left(N_{2} r_{2}\right)}^{(1)}$ :

$$
\mathbf{Y}_{N_{1} \times\left(N_{2} r_{2}\right)}=\mathbf{X}_{N_{1} \times N_{2}} \cdot \mathbf{F}_{N_{2} \times\left(N_{2} r_{2}\right)}^{(1)},
$$

where

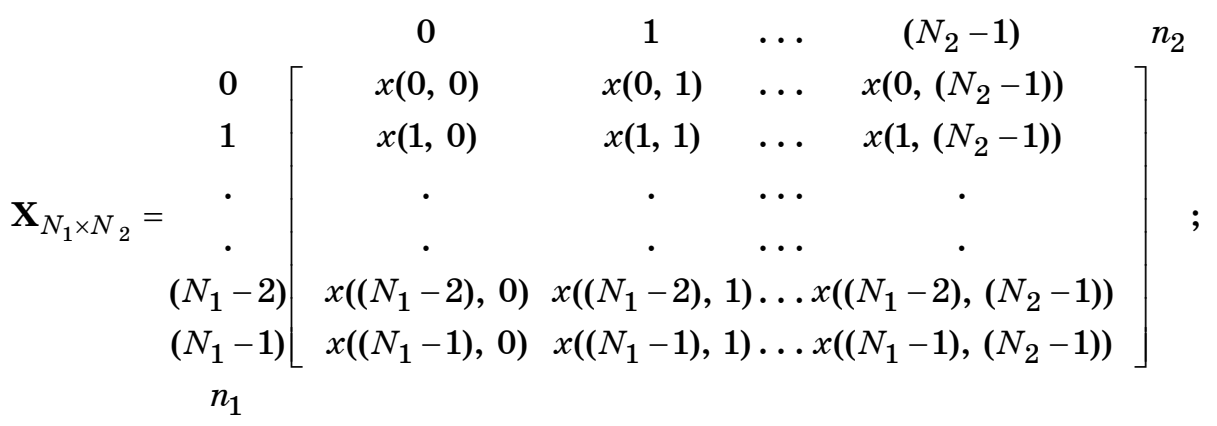

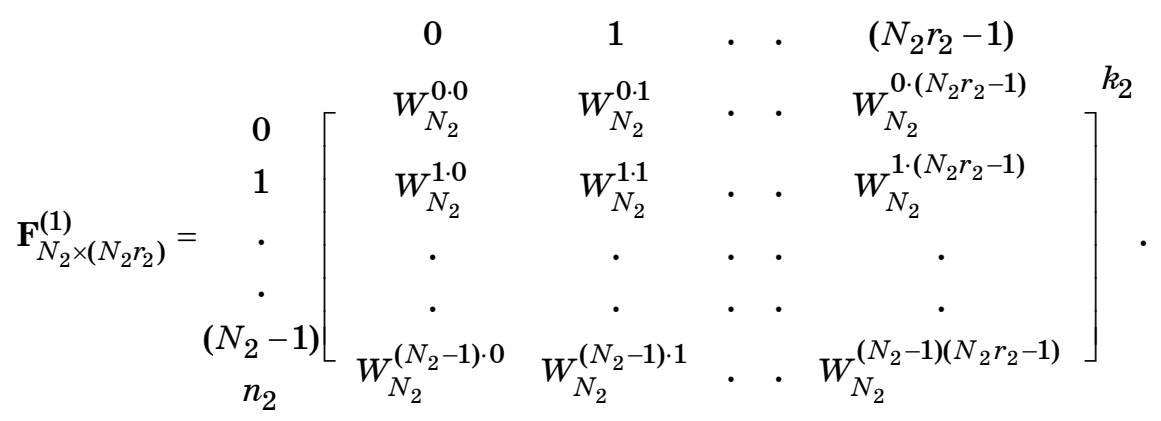

Comparing matrices $\mathbf{F}_{\left(N_{2} r_{2}\right) \times\left(N_{2} r_{2}\right)}^{(1)}(3)$ and $\mathbf{F}_{N_{2} \times\left(N_{2} r_{2}\right)}^{(1)}(5)$, we may see that the matrix $\mathbf{F}_{N_{2} \times\left(N_{2} r_{2}\right)}^{(1)}$ is the result of truncating $\mathrm{N}_{2}\left(r_{2}-1\right)$ the rows of the matrix $\mathbf{F}_{\left(N_{2} r_{2}\right) \times\left(N_{2} r_{2}\right)}^{(1)}$. According to [22], "we denote the set of matrix column numbers by $A$...:

$$
A: A=\left\{0,1,2, \ldots,\left(N_{2} r_{1}-1\right)\right\}
$$


We apply to the set $A$ the relation of comparability modulo $r_{2}$.

It is known that the relation of comparability in modulus $m$ is an equivalence relation and has the properties of reflexivity, symmetry and transitivity."

Also we know that "the relation of comparability modulo $r_{2}$ divides the set $A$ into $r_{2}$ classes of residues modulo $r_{2}$ :

$$
\begin{gathered}
A_{0}=\left\{0, r_{2}, \ldots,\left(N_{2}-1\right) r_{2}\right\} ; \\
\ldots \ldots \ldots \ldots \ldots \ldots . . . . . . . . \\
A_{\left(r_{2}-1\right)}=\left\{\left(r_{2}-1\right), \ldots,\left(N_{2} r_{2}-1\right)\right\} ; \\
A_{i} \neq \varnothing ; A_{i} \bigcap_{i \neq j} A_{j}=\varnothing ; \bigcup_{i=0}^{r_{1}-1} A_{i}=A .
\end{gathered}
$$

The matrix $\mathbf{F}_{N_{2} \times\left(N_{2} r_{2}\right)}^{(1)}$, applying the partition (6) of the set $A$ into $r_{2}$ residue classes modulo $r_{2}$, can be represented in the form of $r_{2}$ matrices of size $N_{2} \times N_{2}$ " [22]:

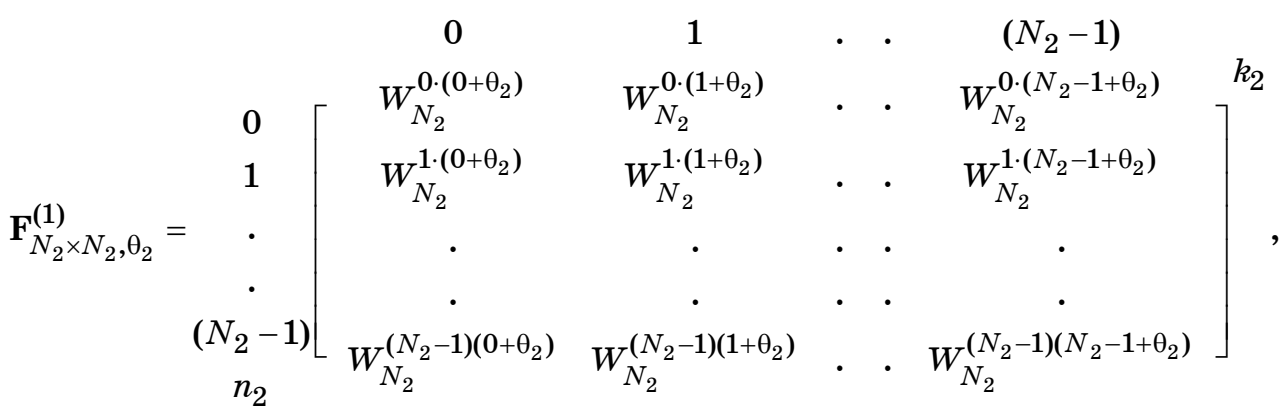

where $\theta_{2}=0 ; 1 / r_{2}, \ldots,\left(r_{2}-1\right) / r_{2}$.

Discrete two-dimensional exponential functions of the form

$$
\begin{gathered}
\operatorname{def}_{H P, N_{1}, N_{2}}\left(k_{1}, n_{1}, k_{2}, n_{2}, \theta_{2}\right)=W_{N_{1}}^{k_{1} n_{1}} \cdot W_{N_{2}}^{\left(k_{2}+\theta_{2}\right) n_{2}}=\left[\exp \left(-j \frac{2 \pi}{N_{1}} k_{1} n_{1}\right)\right] \cdot\left[\exp \left(-j \frac{2 \pi}{N_{2}}\left(k_{2}+\theta_{2}\right) n_{2}\right)\right]= \\
=\left[\cos \left(\frac{2 \pi}{N_{1}} k_{1} n_{1}\right)-j \sin \left(\frac{2 \pi}{N_{1}} k_{1} n_{1}\right)\right] \cdot\left[\cos \left(\frac{2 \pi}{N_{2}}\left(k_{2}+\theta_{2}\right) n_{2}\right)-j \sin \left(\frac{2 \pi}{N_{2}}\left(k_{2}+\theta_{2}\right)\right) n_{2}\right]= \\
=\cos \left(\frac{2 \pi}{N_{1}} k_{1} n_{1}+\frac{2 \pi}{N_{2}}\left(k_{2}+\theta_{2}\right) n_{2}\right)-j \sin \left(\frac{2 \pi}{N_{1}} k_{1} n_{1}+\frac{2 \pi}{N_{2}}\left(k_{2}+\theta_{2}\right) n_{2}\right),
\end{gathered}
$$

where $k_{1}=\overline{0, N_{1}-1} ; \quad k_{2}=\overline{0, N_{2}-1} ; \quad 0 \leq \theta_{2}<1$, will be called two-dimensional discrete exponential functions with a variable parameter $-2 \mathrm{D}$ DEF-VP (Figs. 3-5).
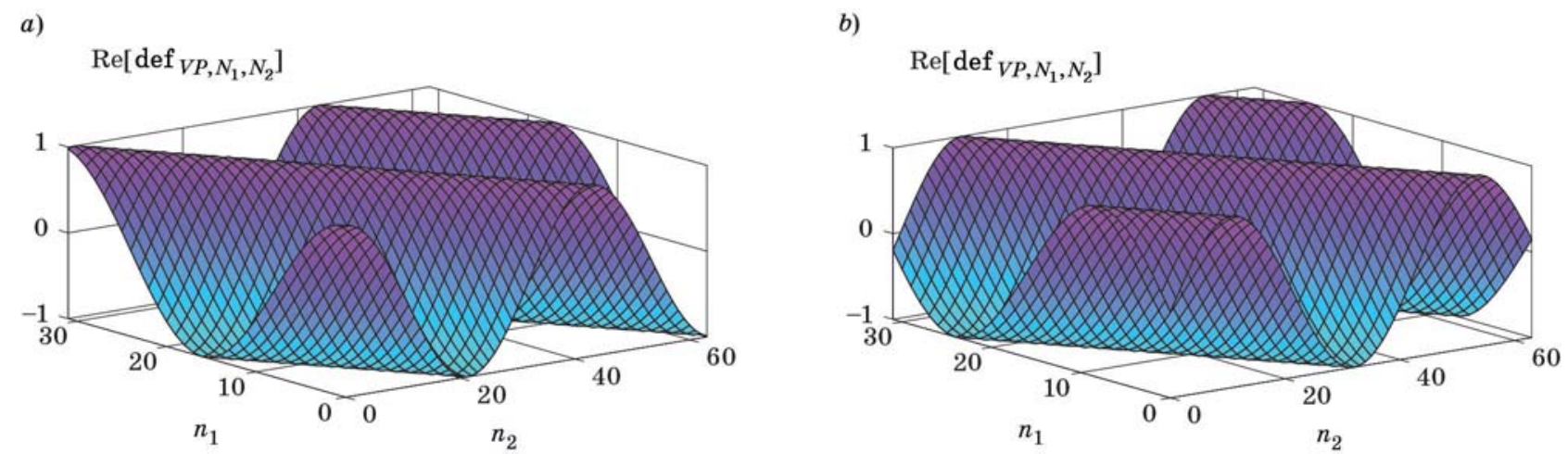

Fig. 3. Two-dimensional exponential function with variable parameter at $N_{1}=32, N_{2}=64 ; k_{1}=1, k_{2}=1 ; \theta_{2}=1 / 2$ : $a$ - a real part; $b$ - an imaginary part 
a)

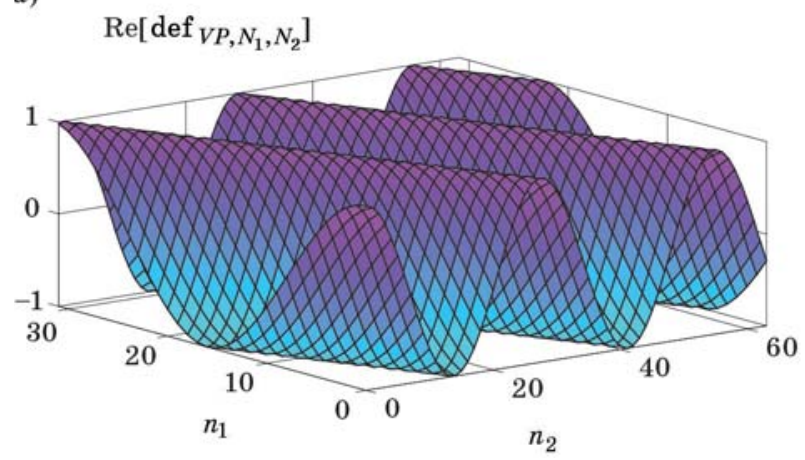

b)

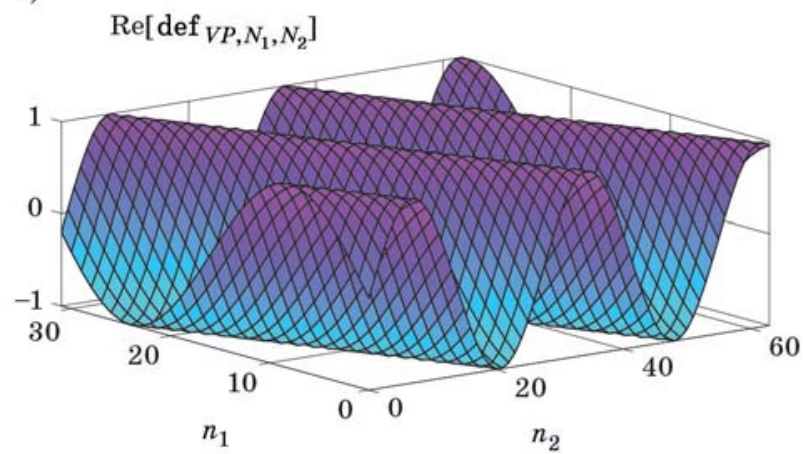

Fig. 4. Two-dimensional exponential function with variable parameter at $N_{1}=32, N_{2}=64 ; k_{1}=1, k_{2}=2 ; \theta_{2}=1 / 3$ : $a$ - a real part; $b$ - an imaginary part
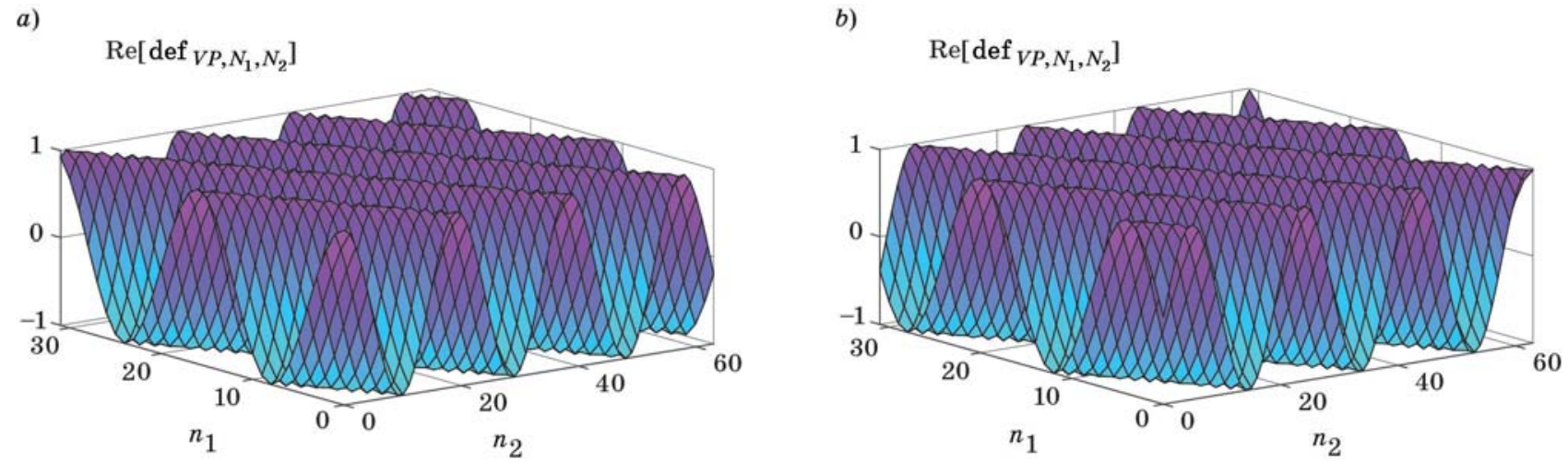

Fig. 5. Two-dimensional exponential function with variable parameter at $N_{1}=32, N_{2}=64 ; k_{1}=2, k_{2}=3 ; \theta_{2}=1 / 3$ : $a$ - a real part; $b$ - an imaginary part

The introduction of discrete exponential functions with a variable parameter makes it possible to generalize the concept of periodicity of the DEF-VP system. Recall that the periodicity of the DEF system in the classical DFT is understood as a periodic continuation of the DEF system outside the interval of $N$ samples. Moreover, the system of discrete basis functions in the classical DFT does not contain discontinuities. In the case of discrete Fourier transform with a variable parameter (DFT-VP) (9), for the DEF-VP system to be inseparable, the periodicity should be understood as parametric periodicity. The parametric periodicity of discrete exponential functions with a variable parameter is understood as their periodic continuation with rotation in complex space by an angle of $2 \pi \theta$. Note that the introduced concept of parametric periodicity is valid for $1 \mathrm{D}$ and $2 \mathrm{D}$ real and complex functions.

Consider the main properties of two-dimensional discrete exponential functions of 2D DEF-VP.

\section{Main properties of 2D DEF-VP}

Each of the two-dimensional discrete exponential functions with a variable parameter has its own spatial frequencies $k_{1}, k_{2}$, which determine its place in a particular basic system. The set of 2D DEF-VP makes its basic system of two-dimensional discrete Fourier transform with a variable parameter (2D DFT-VP) in space $\mathbf{I}_{2}^{N}$.

For each value of the parameter $\theta_{2}$ we can say that:

1. 2D DEF-VP are complex functions by definition.

2. The basis system 2D DEF-VP is a generalization of the basis system 2D DEF and is equal to it at $\theta_{2}=0$.

3. 2D DEF-VP are two-dimensional functions of four equivalent variables $k_{1}, k_{2}, n_{1}, n_{2}$, and one variable parameter $\theta_{2}$ : $\operatorname{def}_{H P, N_{1}, N_{2}}\left(k_{1}, n_{1}, k_{2}, n_{2}, \theta_{2}\right)=W_{N_{1}}^{k_{1} n_{1}} \cdot W_{N_{2}}^{\left(k_{2}+\theta_{2}\right) n_{2}}$.

4. 2D DEF-VP are periodic in variables $k_{1}$ and $n_{1}$ with a period $N_{1}$ and a variable with a period $N_{2}$ :

$$
\begin{gathered}
\operatorname{def}_{H P, N_{1}, N_{2}}\left(\left(k_{1} \pm l N_{1}\right),\left(n_{1}+q N_{1}\right),\left(k_{2} \pm m N_{2}\right),\right. \\
\left.n_{2}, \theta_{2}\right)=\operatorname{def}_{H P, N_{1}, N_{2}}\left(k_{1}, n_{1}, k_{2}, n_{2}, \theta_{2}\right),
\end{gathered}
$$

where $l, m, q$ are integers. 


\section{СИСТЕМНЫЙ АНА^ИЗ}

5. 2D DEF-VP are parametrically periodic in a variable $n_{2}$ with a period $N_{2}$ :

$$
\operatorname{def}_{H P, N_{1}, N_{2}}\left(k_{1}, n_{1}, k_{2}, n_{2} \pm p N_{2}, \theta_{2}\right)=\operatorname{def}_{V P, N_{1}, N_{2}}\left(k_{1}, n_{1}, k_{2}, n_{2}, \theta_{2}\right) \cdot W_{N_{1}}^{\theta_{2} N_{2} p},
$$

where $p$ is integer.

A parametric shift of a two-dimensional signal $X_{N_{1} \times N_{2}}$ in the horizontal direction is understood as a two-dimensional cyclic parametric shift of the form of

$$
\left.\mathbf{C}_{H . S h}=\begin{array}{cc|c}
0 & {\left[\mathbf{X}_{N_{1} \times N_{2}}\right]^{\mathrm{T}} \cdot \mathbf{H}_{H . S h, N_{2} \times N_{2}}^{0}} \\
1 & {\left[\mathbf{X}_{N_{1} \times N_{2}}\right]^{\mathrm{T}} \cdot \mathbf{H}_{H . S h, N_{2} \times N_{2}}^{1}} \\
{\left[\mathbf{X}_{N_{1} \times N_{2}}\right]^{\mathrm{T}} \cdot \mathbf{H}_{H . S h, N_{2} \times N_{2}}^{2}} \\
\cdot \\
\cdot \\
\cdot \\
\cdot \\
\cdot \\
{\left[N_{2}-1\right)} \\
{\left[\mathbf{X}_{N_{1} \times N_{2}}\right]^{\mathrm{T}} \cdot \mathbf{H}_{H . S h, N_{2} \times N_{2}}^{\left(N_{2}-1\right)}}
\end{array}\right],
$$

where $\mathbf{H}_{H . S h, N_{2} \times N_{2}}^{0}$ is two-dimensional identity matrix, expression $\mathbf{H}_{H . S h, N_{2} \times N_{2}}^{m}, m=\overline{\mathbf{0 ,}\left(N_{2}-\mathbf{1}\right)}$ means raising to the power $m$ of the matrix of two-dimensional parametric shift:

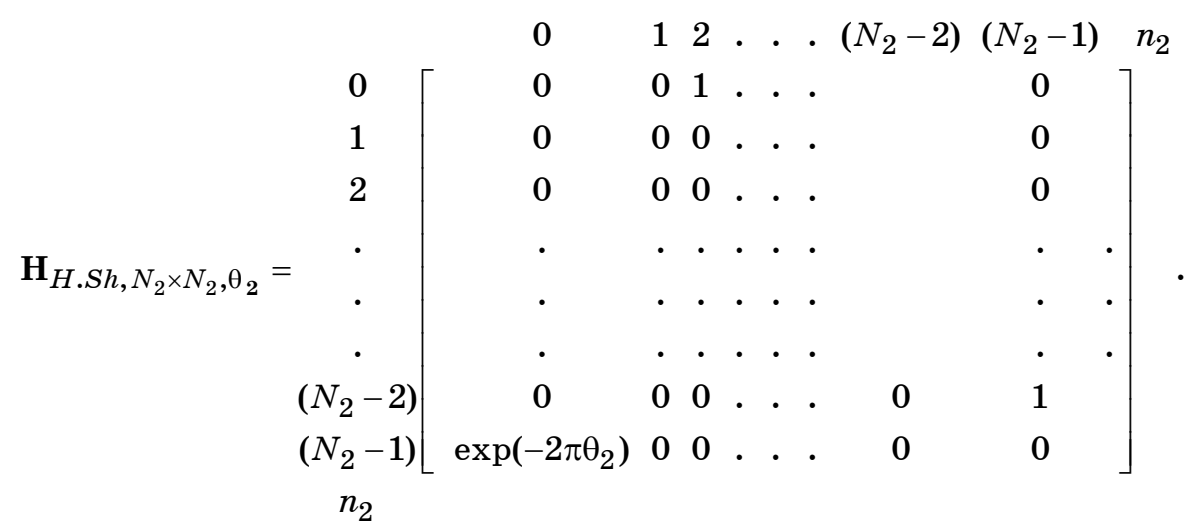

6. The basis system 2D DEF-VP in the variables $k_{1}, k_{2}$ is not multiplicative:

$$
\begin{aligned}
& \operatorname{def}_{H P, N_{1}, N_{2}}\left(k_{1}, n_{1}, k_{2}, n_{2}, \theta_{2}\right) \cdot \operatorname{def}_{H P, N_{1}, N_{2}}\left(k_{3}, n_{1}, k_{4}, n_{2}, \theta_{2}\right) \neq \\
& \quad \neq \operatorname{def}_{H P, N_{1}, N_{2}}\left(\left(k_{1}+k_{3}\right)_{\bmod N_{1}}, n_{1},\left(k_{2}+k_{4}\right)_{\bmod N_{2}}, n_{2}, \theta_{2}\right) .
\end{aligned}
$$

7. The basis system 2D DEF-VP in the variables $n_{1}, n_{2}$ is multiplicative:

$$
\begin{gathered}
\operatorname{def}_{H P, N_{1}, N_{2}}\left(k_{1}, n_{1}, k_{2}, n_{2}, \theta_{2}\right) \cdot \operatorname{def}_{H, N_{1}, N_{2}}\left(k_{1}, n_{3}, k_{2}, n_{4}, \theta_{2}\right)= \\
\quad=\operatorname{def}_{H P, N_{1}, N_{2}}\left(k_{1},\left(n_{1}+n_{3}\right)_{\bmod N_{1}}, k_{2},\left(n_{2}+n_{4}\right)_{\bmod N_{2}}, \theta_{2}\right) .
\end{gathered}
$$

8. Average value of $2 \mathrm{D}$ DEF-IP with spatial frequencies $k_{1} \neq 0, k_{2} \neq 0$ is equal to zero:

$$
\begin{gathered}
\sum_{n_{1}=0}^{N_{1}-1} \sum_{n_{2}=0}^{N_{2}-1} \operatorname{def}_{H P, N_{1}, N_{2}}\left(k_{1}, n_{1}, k_{2}, n_{2}, \theta_{2}\right)=\sum_{n_{1}=0}^{N_{1}-1} W_{N_{1} \times N_{2}}^{N_{2} k_{1} n_{1}}\left[\sum_{n_{2}=0}^{N_{2}-1} W_{N_{1} \times N_{2}}^{N_{2}\left(k_{2}+\theta_{1}\right) n_{2}}\right]= \\
=\left[\frac{1-W_{N_{1}}^{k_{1} N_{1}}}{1-W_{N_{1}}^{k_{1}}}\right]\left[\frac{1-W_{N_{2}}^{\left(k_{2}+\theta_{2}\right) N_{2}}}{1-W_{N_{2}}^{k_{2}}}\right]=0 .
\end{gathered}
$$


9. The basic system 2D DEF-VP is an orthogonal basis system with respect to variables $k_{1}, k_{2}$ :

$$
\begin{gathered}
\frac{1}{N_{1} N_{2}} \sum_{n_{1}=0}^{N_{1}-1} \sum_{n_{2}=0}^{N_{2}-1} W_{N_{1} \times N_{2}}^{\left(N_{2} k_{1} n_{1}+N_{1}\left(k_{2}+\theta_{2}\right) n_{2}\right)} \times \\
\times W_{N_{1} \times N_{2}}^{\left(N_{2} k_{3} n_{1}+N_{1}\left(k_{4}+\theta_{2}\right) n_{2}\right)^{*}}=\left\{\begin{array}{l}
1, \text { if } k_{1}=k_{3}, k_{2}=k_{4} \\
0, \text { if } k_{1} \neq k_{3}, k_{2} \neq k_{4}
\end{array} ;\right.
\end{gathered}
$$

and with respect to variables $n_{1}, n_{2}$ :

$$
\begin{gathered}
\frac{1}{N_{1} N_{2}} \sum_{k_{1}=0}^{N_{1}-1} \sum_{k_{2}=0}^{N_{2}-1} W_{N_{1} \times N_{2}}^{\left(N_{2} k_{1} n_{1}+N_{1}\left(k_{2}+\theta_{2}\right) n_{2}\right)} \times \\
\times W_{N_{1} \times N_{2}}^{\left(N_{2} k_{1} n_{3}+N_{1}\left(k_{2}+\theta_{2}\right) n_{4}\right)^{*}}=\left\{\begin{array}{l}
1, \text { if } n_{1}=n_{3}, n_{2}=n_{4} \\
0, \text { if } n_{1} \neq n_{3}, n_{2} \neq n_{4}
\end{array},\right.
\end{gathered}
$$

where the symbol * means complex conjugation.

10. 2D DEF-VP can be represented by two unit vectors, which represent $W_{N_{1}}^{k_{1} n_{1}}$ and $W_{N_{2}}^{\left(k_{2}+\theta_{2}\right) n_{2}}$. The unit vectors rotate discontinuously (discretely) in perpendicular complex planes. On the interval $N_{1}$, the unit vector which displays $W_{N_{1}}^{k_{1} n_{1}}$, passes the angle of $2 \pi k_{1}$ radians, making $k_{1}$ revolutions, and on the interval $N_{2}$, the unit vector, representing $W_{N_{2}}^{\left(k_{2}+\theta_{2}\right) n_{2}}$, passes the angle $2 \pi\left(k_{2}+\theta_{2}\right)$ radians, making $\left(k_{2}+\theta_{2}\right)$ revolutions. The unit vectors representing the complex conjugate DEF-VP:

$$
\begin{gathered}
W_{N_{1}}^{-k_{1} n_{1}}=W_{N_{1}}^{\left(N_{1}-k_{1}\right) n_{1}} \text { and } \\
W_{N_{2}}^{-\left(k_{2}+\theta_{2}\right) n_{2}}=W_{N_{2}}^{\left(N_{2}-\left(k_{2}+\theta_{2}\right)\right) n_{2}}
\end{gathered}
$$

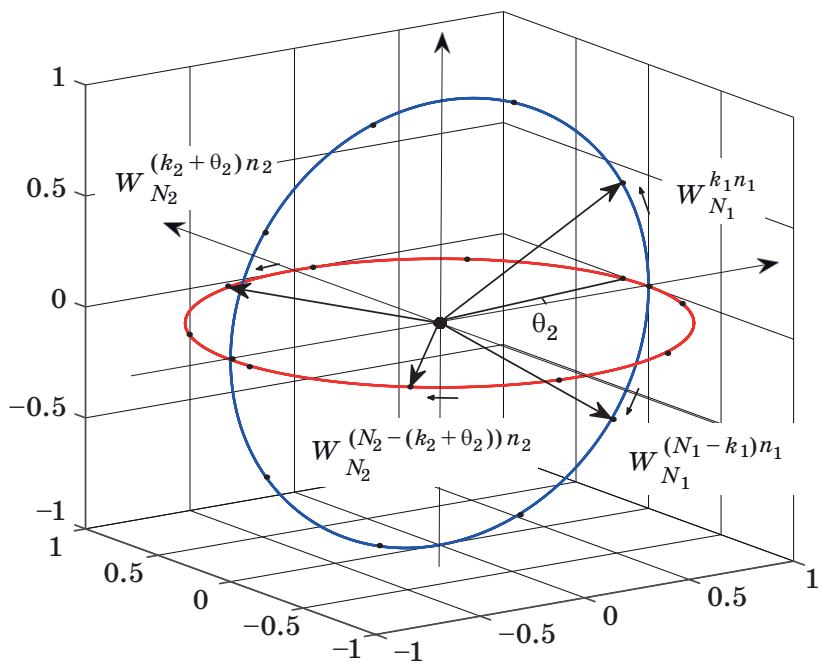

- Fig. 6. Representation of a two-dimensional discrete exponential function with a variable parameter in the spatial domain and make $\left(N_{1}-k_{1}\right)$ and $\left(N_{2}-\left(k_{2}+\theta_{2}\right)\right)$ revolutions respectively.

Figure 6 illustrates such a 2D DEF-VP representation, where the angles $2 \pi k_{1} / N_{1}$ and $2 \pi\left(k_{2}+\theta_{2}\right) / N_{2}$ are marked with the corresponding points.

11. The basis system 2D DEF-VP is complete in space $\mathbf{I}_{2}^{N}$.

Expansion in basis systems of the form (8) is defined as a 2D DFT-VP. Algebraic form of 2D DFT-VP

$$
\begin{gathered}
S_{N_{1}, N_{2}}\left(k_{1}, k_{2}, \theta_{2}\right)= \\
=\frac{1}{N_{1} N_{2}} \sum_{n_{1}=0}^{N_{1}-1} \sum_{n_{2}=0}^{N_{2}-1} x\left(n_{1}, n_{2}\right) W_{N_{1}}^{k_{1} n_{1}} \cdot W_{N_{2}}^{\left(k_{2}+\theta_{2}\right) n_{2}},
\end{gathered}
$$

where $k_{1}, k_{2}$ are spatial frequencies, $k_{1}=\overline{0,\left(N_{1}-1\right)}$, $k_{2}=\overline{\mathbf{0 ,}\left(N_{2}-1\right)} ; \theta_{2}$ is a parameter, $0 \leq \theta_{2}<1 ; x\left(n_{1}\right.$, $\left.n_{2}\right)$ - two-dimensional signal, $n_{1}=\overline{0, N_{1}-1}$, $n_{2}=\overline{0, N_{2}-1} ; \quad S_{N_{1}, N_{2}}\left(k_{1}, k_{2}, \theta_{2}\right)$ are bins of $2 \mathrm{D}$ DFT-VP (two-dimensional vector spatial-frequency spectrum of the signal $x\left(n_{1}, n_{2}\right)$ in the basic 2D DEFVP system).

The algebraic form of direct 2D DFT-VP, taking into account the property of separability of the kernel (core) of 2D DFT-VP, can be represented as

$$
\begin{gathered}
S_{N_{1}, N_{2}}\left(k_{1}, k_{2}, \theta_{2}\right)= \\
=\frac{1}{N_{1}} \sum_{n_{1}=0}^{N_{1}-1} W_{N_{1}}^{k_{1} n_{1}}\left[\frac{1}{N_{2}} \sum_{n_{2}=0}^{N_{2}-1} x\left(n_{1}, n_{2}\right) W_{N_{2}}^{\left(k_{2}+\theta_{2}\right) n_{2}}\right] .
\end{gathered}
$$

It can be seen that formula (10) makes it possible to step-by-step calculation of the direct $2 \mathrm{D}$ DFT-VP by the method of sequential calculation of two DFT-P (parametric DFT). Note that the calculation of the DFT-P can be carried out by methods of parametric fast Fourier transform (FFT-P) [1].

There is an inverse 2D DFT-VP (2D IDFT-VP):

$$
\begin{gathered}
x\left(n_{1}, n_{2}\right)= \\
=\sum_{k_{1}=0}^{N_{1}-1} \sum_{k_{2}=0}^{N_{2}-1} S_{N_{1}, N_{2}}\left(k_{1}, k_{2}, \theta_{2}\right) W_{N_{1}}^{-k_{1} n_{1}} \cdot W_{N_{2}}^{-\left(k_{2}+\theta_{2}\right) n_{2}},
\end{gathered}
$$

where $n_{1}=\overline{0, N_{1}-1} ; n_{2}=\overline{0, N_{2}-1}$.

Using the separability property of the 2D DFTVP kernel, we can introduce the matrix form of the direct 2D DFT-VP:

$$
\mathbf{S}_{N_{1} \times N_{2}, \theta_{2}}=\frac{1}{N_{1}} \mathbf{F}_{N_{1} \times N_{1}}^{(2)} \cdot \frac{1}{N_{2}}\left[\mathbf{X}_{N_{1} \times N_{2}} \cdot \mathbf{F}_{N_{2} \times N_{2}, \theta_{2}}^{(1)}\right],
$$

where $0 \leq \theta_{2}<1$; 


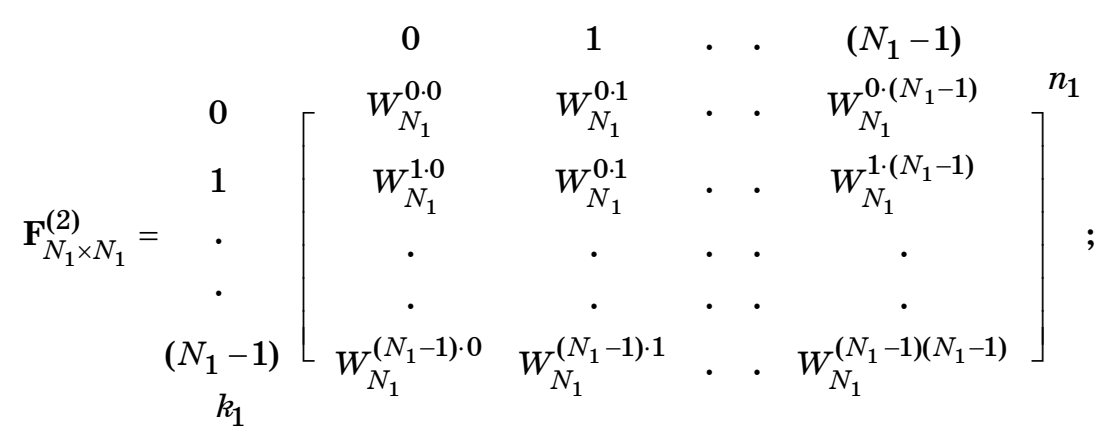

$\mathbf{X}_{N_{1} \times N_{2}}$ is given by relation (4);

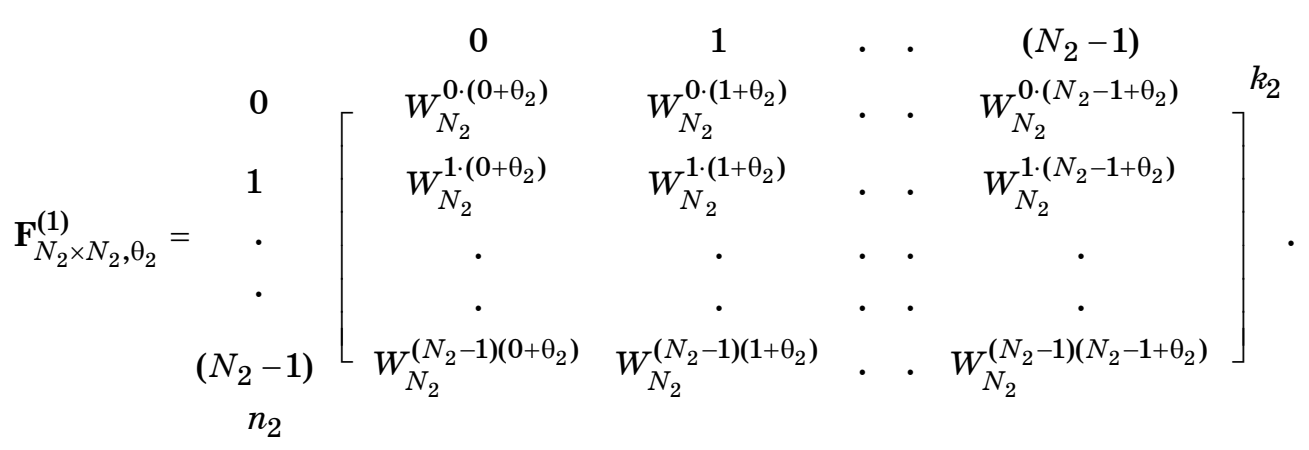

We note the difference between matrices (11) and (7), which lies in the nature of the parameter $\theta_{2}$ change. The inverse 2D DFT-VP in matrix form is determined by the matrix equation

$$
\mathbf{X}_{N_{1} \times N_{2}}=\frac{1}{N_{1}} \mathbf{F}_{N_{1} \times N_{1}}^{(2) *} \cdot \frac{1}{N_{2}}\left[\mathbf{S}_{N_{1} \times N_{2}, \theta_{2}} \cdot \mathbf{F}_{N_{2} \times N_{2}, \theta_{2}}^{(1) *}\right]
$$

where $0 \leq \theta_{2}<1$.

It can be shown that the theorems of linearity, shift, correlation and Parseval's equality are valid for 2D DFT-VP. For 2D DFT-VP, similar to 2D DFT, the concepts of power spectrum $P_{N_{1}, N_{2}}\left(k_{1}, k_{2}, \theta_{2}\right)$ and energy spectrum $G_{N_{1}, N_{2}}\left(k_{1}, k_{2}, \theta_{2}\right)$ can be introduced

$$
P_{N_{1}, N_{2}}\left(k_{1}, k_{2}, \theta_{2}\right)=\left|S_{N_{1}, N_{2}}\left(k_{1}, k_{2}, \theta_{2}\right)\right|^{2} ; G_{N_{1}, N_{2}}\left(k_{1}, k_{2}, \theta_{2}\right)=P_{N_{1}, N_{2}}\left(k_{1}, k_{2}, \theta_{2}\right) / \Delta f ; \Delta f=1 /\left(N_{1} N_{2}\right) .
$$

Let us estimate the efficiency of increasing the detailing of the two-dimensional energy spectrum $G_{N_{1}, N_{2}}\left(k_{1}, k_{2}\right)$ using 2D DFT-VP in comparison with the classical 2D DFT.

\section{Evaluation of the efficiency of Fourier-processing of signals padded with zero samples in 2D DFT-VP basis}

The increase in the detailing of the two-dimensional energy spectrum $G_{N_{1}, N_{2}}\left(k_{1}, k_{2}\right)$ by $r_{2}$ times is carried out by padding the horizontal period of the 2 D signal $X_{N_{1} \times N_{2}}$ with $\left(r_{2}-1\right)$ zero matrices $\mathbf{O}_{N_{1} \times N_{2}}$ (1). Padding the horizontal period of a 2D signal $X_{N_{1} \times N_{2}}$ with $\left(r_{2}-1\right)$ zero matrices $\mathbf{O}_{N_{1} \times N_{2}}$ makes it possible to obtain a new 2D signal $X_{N_{1} \times\left(N_{2} r_{2}\right)}$ from a 2D signal $X_{N_{1} \times N_{2}}$.

Applying the 2D DFT in algebraic form to the 2D signal $X_{N_{1} \times\left(N_{2} r_{2}\right)}$, we obtain the number of coefficients (bins) of 2D DFT $S_{N_{1}, N_{2} r_{2}}\left(k_{1}, k_{2}\right)$, which is $r_{2}$ times greater than with 2D DFT of the signal $X_{N_{1} \times N_{2}}$. However, obtaining a $r_{2}$ times more detailed energy spectrum $G_{N_{1}, N_{2} r_{2}}\left(k_{1}, k_{2}\right)$ by a method based on the separability of the 2D DFT kernel, will require additional $\left(r_{2}-1\right) N_{1} N_{2}$ cells for storing zero samples and implementing $N_{1} N_{2} r_{2}\left(N_{1}+N_{2} r_{2}\right)$ additional complex operations.

Obtaining an $r_{2}$ times detailed energy spectrum $G_{N_{1}, N_{2} r_{2}}\left(k_{1}, k_{2}\right)$ by a method based on the separability property of the 2D DFT-VP kernel does not require additional RAM (storage) for storing zero samples and requires $N_{1} N_{2} r_{2}\left(N_{1}+N_{2}\right)$ complex operations. Thus, the use of 2D DFT-VP instead of the classic 2D DFT allows:

- decrease number of complex operations by $\gamma=\frac{N_{1}+N_{2} r_{2}}{N_{1}+N_{2}}$ times; 
- decrease storage size by $r_{2}$ times;

- parallelize the process of detailing the two-dimensional energy spectrum $G_{N_{1}, N_{2}}\left(k_{1}, k_{2}\right)$, thus reducing the execution time of the $2 \mathrm{D}$ DFT by $r_{2}$ times.

\section{Conclusions}

Discrete Fourier transforms with a variable parameter have been developed. These transforms make it possible to efficiently process two-dimensional signals, the horizontal periods of which are padded with zero samples. The generalization of classical two-dimensional discrete Fourier transform is based on a new method of splitting the rectangular matrix of discrete Fourier transform into square matrices. The splitting of rectangular matrices into square matrices is carried out by using the ordering of the columns of rectangular matrices using the equivalence relation - the relation of comparability in modulus. The properties of the bases of the proposed transformations are investigated. The valid- ity for Fourier transforms with variable parameters of the following theorems is proved: linearity, shift, correlation, and Parseval's equality.

New concepts of digital spectral Fourier analysis are introduced: the concept of parametric shift of two-dimensional signal and parametric periodicity of two-dimensional signal. The estimation of the reducing the amount of RAM (random access memory) needed and the number of calculations when applying the proposed transforms is carried out in comparison with the application of classical two-dimensional discrete Fourier transform to 2D signals padded with zero samples. Developed two-dimensional discrete Fourier transform with variable parameters can significantly reduce the cost of Fourier-processing of two-dimensional signals (including images), padded with zero samples. In addition, the developed transforms also allow parallelizing the process, thus significantly reducing Fourier-processing time. Note one more application of developed two-dimensional discrete Fourier transform with variable parameters: determination of the parameters of $2 \mathrm{D}$ hidden periodicities by varying the parameter $\theta_{2}$.

\section{References}

1. Ponomarev A. V. Systems Analysis of Discrete Two-Dimensional Signal Processing in Fourier Basis. In: Advances in Signal Processing. Theories, Algorithms, and System Control-7. Favorskaya M. N., Jain L. C. (eds). Springer, Cham. Vol. 184. Pp. 87-96. doi.org/10/1007/978-3-030-40312-6_7

2. Ponomareva O. V., Ponomarev A. V., Smirnova N. V. Sliding Spatial Frequency Processing of Discrete Signals. In: Advances in Signal Processing. Theories, Algorithms, and System Control-8. Favorskaya M. N., Jain L. C. (eds). Springer, Cham. Vol. 184. Pp. 97-110. doi.org/10/1007/978-3-030-40312-6_8

3. Favorskaya M., Savchina E., Popov A. Adaptive visible image watermarking based on Hadamard transform. IOP Conference Series: Materials Science and Engineering, 2018, vol. 450, no. 5, MIST Aerospace, pp. 052003.1-052003.6. doi:10.1088/1757-899X/450/ $5 / 052003$

4. Klionskiy D. M., Kaplun D. I., Geppener V. V. Empirical more decomposition for signal preprocessing and classification of intrinsic mode functions. Pattern Recognition and Image Analysis (Advances in Mathematical Theory and Applications), 2018, vol. 28, no. 1, pp. 122-132. doi:10.1134/S1054661818010091

5. Batishchev V. I., Volkov I. I., Zolin A. G. Using a stochastic basis in signal and image recovery problems. Optoelectronics, Instrumentation and Data Processing, 2017, vol. 53, no. 4, pp. 414-420.

6. Bakulin M. G., Vityazev V. V., Shumov A. P., Kreyndelin V. B. Effective signal detection for the spatial multiplexing mimo systems. Telecommunications and $R a$ - dio Engineering, 2018. vol. 77, no. 13, pp. 1141-1158. doi.org/10.1615/TelecomRadEng.v77.i13.30

7. Lerner I. M., Il'In G. I., Il'In V. I. To the matter of optimal transfer characteristics of linear selective systems of communication channel with memory and apsk-n. 2019 Systems of Signal Synchronization, Generating and Processing in Telecommunications, Synchroinfo, 2019, pp. 8814277. doi.org/10.1109/SYNCHROINFO.2019.8814021

8. Kulikovskikh I., Prokhorov S. Psychological perspectives on implicit regularization: a model of retrieval-induced forgetting (RIF). Journal of Physics: Conference Series, 2018, pp. 012079. doi:10.1088/1742$6596 / 1096 / 1 / 012079$

9. Lysenko N., Labkov G. Applying of Kutter-Jor-danBossen steganographic algorithm in video sequences. 2017 IEEE Conference of Russian Young Researchers in Electrical and Electronic Engineering (EIConRus), 2017,pp.695-696.doi:10.1109/EICon-Rus.2017.7910651

10. Favorskaya M. N., Buryachenko V. V. Authentication and Copyright Protection of Videos under Transmitting Specifications. In: Computer Vision in Advanced Control Systems-5. Favorskaya M. N., Jain L. C. (eds). Springer, Cham, 2020. Vol. 175. Pp. 119-160. doi. org/10.1007/978-3-030-33795-7_5

11. Khanyan G. S. Sampling theorem in frequency domain for the finite spectrum. Proceedings of 2018 IEEE EastWest Design and Test Symposium, EWDTS 2018, 2018, pp. 8524822. doi:10.1109/EWDTS.2018.8524822

12. Petrovsky N. A., Rybenkov E. V., Petrovsky A. A. Two-dimensional non-separable quaternionic paraunitary filter banks. 22nd IEEE Signal Processing: Algorithms, Architectures, Arrangements, and Applica- 
tions, SPA 2018, 2018, pp. 120-125. doi:10.23919/ SPA.2018.8563311

13. Likhttsinder B. Conditional average value of queues in queuing systems with bath request flows. 2017 th International Scientific-Practical Conference Problems of Infocommunications Science and Technology, PIC S and T 2017 - Proceedings, 2018, pp. 49-52. doi:10.1109/INFOCOMMST.2017.8246347

14. Khanyan G. S. Frequency domain sampling theorem for an infinite spectrum. DSPA: Voprosy primeneniya cifrovoj obrabotki signalov, 2018, vol. 8, no. 2, pp. 5661 (In Russian).

15. Bakulin M. G., Vityazev V. V., Shumov A. P., Kreyndelin V. B. Effective signal detection for the spatial multiplexing mimo systems. Telecommunications and Radio Engineering, 2018. vol. 77, no. 13, pp. 1141-1158. doi.org/10.1615/TelecomRadEng.v77.i13.30

16. Smirnova N. V., Ponomareva O. V. Vector and spectral digital signal processing in musical acoustics using the parametric discrete Fourier transform. Digital Signal Processing, 2019, no. 2, pp. 3-11 (In Russian).

17. Klionskiy D. M., Geppener V. V. Application of iterative procedures for signal processing. Proceedings of the 2018 IEEE Conference of Russian Young Researchers in Electrical and Electronic Engineering, ElConRus 2018, 2018, pp. 1090-1093. doi:10.1109/EIConRus.2018.8317280

18. Bakulin M. G., Vityazev V. V., Shumov A. P., Kreyndelin V. B. Effective signal detection for the spatial mul- tiplexing mimo systems. Telecommunications and $R a$ dio Engineering, 2018, vol. 77, no. 13, pp. 1141-1158.

19. Prozorov D., Tatarinova A. Comparison of grapheme-to-phoneme conversions for spoken document. 2019 IEEE East-West Design and Test Symposium, EWDTS 2019, 2019, pp. 8884449. doi:10.1109/EWDTS.2019.8884449

20. Prozorov D., Trubin I. Detection of a signal in the simo system with spatial correlation of noise. 2018 Proceedings of 7th Mediterranean Conference on Embedded Computing, MECO 2018 - Including ECYPS 2018, 2018, pp. 1-5. doi:10.1109/MECO.2018.8405965

21. Urakov A., Gurevich K., Alies M., Reshetnikov A., Kasatkin A., Urakova N. The tissue temperature during injection of drug solution into it as an integral indicator of rheology. Journal of Physics: Conference Series. 4th International Conference on Rheology and Modeling of Materials, IC-RMM 2019, 2020, vol. 1527, pp. 012003. doi:10.1088/1742-6596/1527/1/012003

22. Ponomarev A. V. Fundamentals of the theory of two-dimensional digital signal processing in Fourier bases with variable parameters. Digital Signal Processing, 2019, no. 2, pp. 12-20 (In Russian).

23. Ponomareva N. V. Problems of computer spectral signal processing in musical acoustics. Intelligent Systems in Manufacturing, 2018, vol. 16, no. 1, pp. 26-33 (In Russian). doi:10.22213/24-10-9304-2018-1-26-33

УДК 621.372

doi:10.31799/1684-8853-2021-1-55-65

Теоретические основы цифрового векторного фурье-анализа двумерных сигналов, дополненных нулевыми отсчетами

О. В. Пономарева ${ }^{a}$, доктор техн. наук, профессор, orcid. org/0000-0002-7311-3108, ponva@mail.ru

А. В. Пономарев ${ }^{\mathrm{a}}$, канд. экон. наук, доцент, orcid.org/0000-0002-3746-9289

аИжевский государственный технический университет им. М. Т. Калашникова, Студенческая ул., 7, Ижевск, 426069, РФ

Введение: практика применения фурье-обработки финитных двумерных сигналов (в том числе изображений), подтвердив ее действенность, выявила и ряд присущих ей отрицательных эффектов. Известный метод борьбы с негативными эффектами фурье-обработки - операция дополнения сигналов нулями. Однако применение этой операции приводит к необходимости обеспечения информационно-управляющих систем дополнительной памятью и проведения ими непроизводительных вычислений. Цель: разработка новых дискретных преобразований Фурье для эффективной и результативной обработки двумерных сигналов, дополненных нулями. Методы: предложен новый метод разбивки прямоугольной матрицы дискретного преобразования Фурье на квадратные матрицы. Метод основан на применении для упорядочения строк (столбцов) матрицы Фурье отношения сравнимости по модулю. Результаты: разработаны новые дискретные преобразования Фурье с варьируемыми параметрами, являющиеся обобщением классического дискретного преобразования Фурье. Исследованы свойства базисов преобразований Фурье с варьируемыми параметрами. Для данных преобразований доказаны теоремы линейности, сдвига, корреляции и равенство Парсеваля. В цифровой спектральный фурье-анализ введены понятия параметрического сдвига двумерного сигнала и параметрической периодичности двумерного сигнала. Выполнена оценка сокращения требуемого объема памяти и числа вычислений в случае применения предложенных преобразований. Проведено их сравнение с дискретным преобразованием Фурье. Практическая значимость: разработанные дискретные преобразования Фурье с варьируемыми параметрами позволяют существенно сократить затраты на фурье-обработку двумерных сигналов (в том числе изображений), дополненных нулями.

Ключевые слова - дискретное преобразование Фурье, двумерный сигнал, фурье-обработка, эффекты дискретного преобразования Фурье, базис, варьируемый параметр.

Для цитирования: Ponomareva O. V., Ponomarev A. V. Theoretical foundations of digital vector Fourier analysis of two-dimensional signals padded with zero samples. Инфорлационно-управляюшие систелы, 2021, № 1, с. 55-65. doi:10.31799/1684-8853-2021-1-55-65 For citation: Ponomareva O. V., Ponomarev A. V. Theoretical foundations of digital vector Fourier analysis of two-dimensional signals padded with zero samples. Informatsionno-upravliaiushchie sistemy [Information and Control Systems], 2021, no. 1, pp. 55-65. doi:10.31799/1684-8853-2021-1-55-65 